\title{
Evaluation of morphological and functional nerve recovery of rat sciatic nerve with a Hyaff11-based nerve guide
}

doi:10.1533/abbi.2005.0017

\author{
K. Jansen ${ }^{1}$, Y. Ludwig², M. J. A. van Luyn², \\ A. A. Gramsbergen ${ }^{3}$ and M. F. Meek ${ }^{2}$ \\ ${ }^{1}$ Hutchinson Technology Inc., Ifsselburcht 3, Arnhem, The Netherlands \\ ${ }^{2}$ Tissue Engineering, Medical Biology, Department of Pathology Lab Medicine, Faculty of Medical Sciences, \\ University of Groningen, Hanzeplein 1, 9713 GZ Groningen, The Netherlands \\ ${ }^{3}$ Department of Medical Physiology, University of Groningen, A. Deusinglaan 1, 9713 AV, Groningen, \\ The Netherlands
}

\begin{abstract}
Application of a Hyaff11-based nerve guide was studied in rats. Functional tests were performed to study motor nerve recovery. A withdrawal reflex test was performed to test sensory recovery. Morphology was studied by means of histology on explanted tissue samples. Motor nerve recovery was established within 7 weeks. Hereafter, some behavioral parameters like alternating steps showed an increase in occurence, while others remained stable. Sensory function was observed within the 7 weeks time frame. Nerve tissue had bridged the $10-\mathrm{mm}$ gap within 7 weeks. The average nerve fiber surface area increased significantly in time. In situ degradation of the nerve conduit was fully going on at week 7 and tubes had collapsed by then. At weeks 15 and 21, the knitted tube wall structure was completely surrounded by macrophages and giant cells, and matrix was penetrating the tube wall. We conclude that a Hyaff11-based nerve guide can be used to bridge short peripheral nerve defects in rat. However, adaptations need to be made.
\end{abstract}

Key words: Nerve repair, Hyaff11, rat sciatic nerve, morphology, immunohistology, macrophages.

\section{INTRODUCTION}

Esterified hyaluronan derivatives (Hyaff11) promise to be suitable candidates for application in pharmaceutical and medical science (Davidson et al. 1991; Joshi et al. 1992; Galassi et al. 2000; Avitabile et al. 2001). Hyaff11 (with benzyl alcohol $100 \%$ esterified hyaluronan) is a good carrier for keratinocytes to be grafted onto skin wounds (Andreassi 1995; Zacchi et al. 1998), while fibroblasts and chondroblasts can also be grown on Hyaff11 biomaterials (Zacchi et al. 1998; Galassi et al. 2000; Grigolo et al. 2001).

Nowadays, artificial biodegradable nerve guides are widely studied for bridging peripheral nerve defects (Den Dunnen 1996; Doolabh et al. 1996; Meek 2000). It was

\section{Corresponding Author:}

K. Jansen

Hutchinson Technology Inc

IJsselburcht 3, 6825 BS Arnhem, The Netherlands

Tel: 3126 3653371; Fax: 31263653372

Email: koen.jansen@hti.htch.com hypothesised that in such a repair system, hyaluronic acid organises the extra-cellular matrix into a hydrated open lattice during the fibrin matrix phase of regeneration, thereby facilitating migration of the regenerating axons. Also, hyaluronic acid injected in nerve guides enhances peripheral nerve regeneration (Wang et al. 1998; Mohammad et al. 2000). Furthermore, hyaluronic acid is a compound associated with decreased scarring and improved fibrin matrix formation, implying that hyaluronic acid acts as an agent that aids nerve growth and repair (Seckel et al. 1995).

Hyaff11 materials have been the subject of investigation on cytotoxicity and biocompatibility (Benedetti et al. 1993; Campoccia et al. 1996), and earlier we showed that the Hyaff11-based nerve guide has no cytotoxic effects in vitro and shows good biocompatibility/biodegradation in vivo (Jansen et al., unpublished data). In the present study, we examine the application of the Hyaff11-based nerve guide in situ, crossing a $1-\mathrm{cm}$ gap in the rat sciatic nerve. Tissue reactions, in situ biodegradation, nerve fiber restoration, and functional repair were examined at 7,15 and 21 weeks after implantation. 


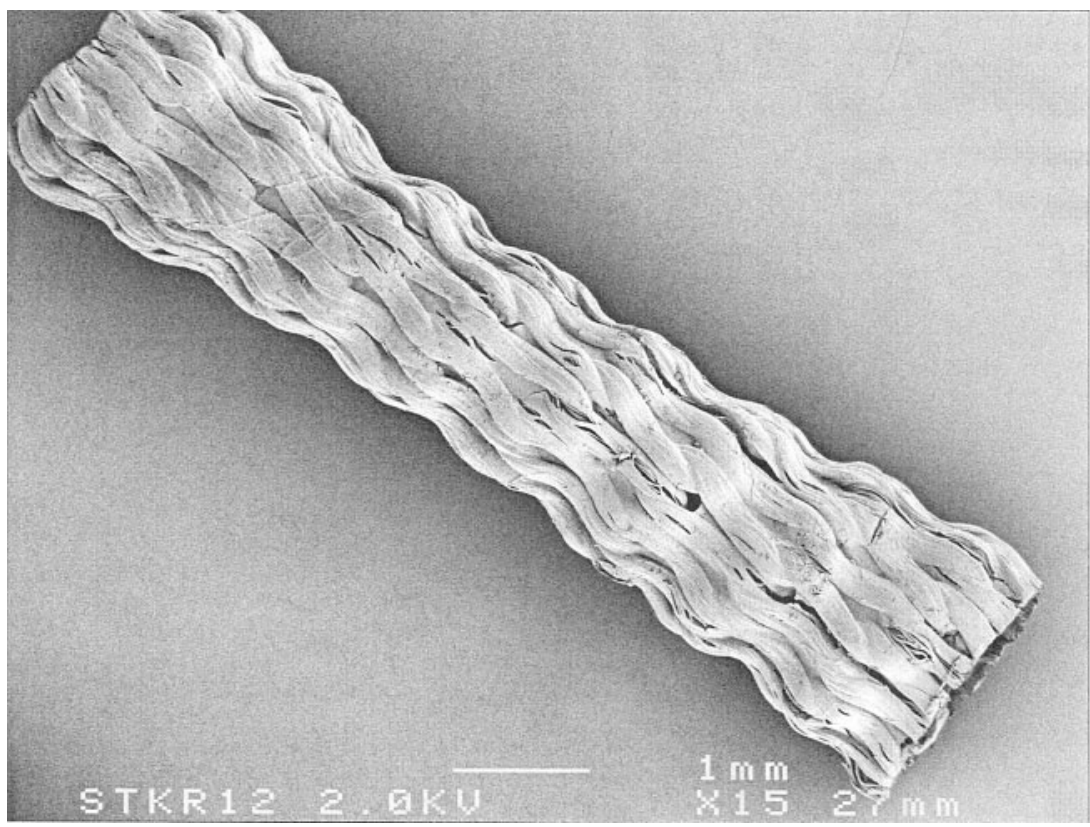

Figure 1 Scanning electron micrograph of the Hyaff11-based nerve guide. The knitted structure is clearly visible. Bar represents $1 \mathrm{~mm}$.

\section{MATERIALS AND METHODS}

\section{Animals}

Male Wistar rats $(n=15)$, weighing approximately $250 \mathrm{~g}$, were purchased from Harlan Nederland BV. Animals were individually housed under controlled conditions for 2 weeks. Prior to conduit implantation and sacrificing, rats were pre-medicated with atropine $(0.25 \mathrm{mg} / \mathrm{kg}$ body weight) and anesthetised with $1 \%$ isoflurane (Forene, $\mathrm{Ab}-$ bott Laboratories Ltd., Queenborough, Kent, UK) and $\mathrm{O}_{2} / \mathrm{N}_{2} \mathrm{O}$.

\section{Conduits}

Hyaff11 conduits were obtained from Fidia Advanced Biopolymers (Abano Terme, Italy). Preparation of the polymer has been described before (Kyyronen et al. 1992; Benedetti et al. 1993). Conduits are prepared from individually knitted strands and are strengthened by coating with a thin layer of the same Hyaff 11 polymer. This resulted in a conduit with an internal diameter of $2 \mathrm{~mm}$, a length of $18 \mathrm{~mm}$ and a wall thickness of $0.2 \mathrm{~mm}$ (Figure 1). Such conduit was then cut to the right length $(12 \mathrm{~mm})$ during the operation procedure.

\section{Operation procedure}

The left sciatic nerve was exposed by splitting the left superficial gluteal muscle. A gap of $10 \mathrm{~mm}$ was made. Continuity was re-established by interposing a $12-\mathrm{mm}$ biodegradable Hyaff11 conduit. Both the proximal and distal ends of the sciatic nerve were telescoped into the ends of the nerve guide and fixed with a single 9-0 nylon suture (Auto Suture Company, Norwalk, CT; MV
100-4 needle). The tube was prefilled with phosphate buffered saline (PBS; NPBI, Emmercompascuum, The Netherlands).

Surgical procedures were performed under an operation microscope (Zeiss OPMI-6, Weesp, The Netherlands), and a sterile procedure was used throughout the operation. After surgery, the animals were housed in a temperatureand humidity-controlled room with 12-h light cycles and had access to standard rat food and water ad libitum. Good laboratory practice was maintained, according to the guidelines of the Dutch Ministry (Veterinary Health Inspection), comparable with the international rules for animal experimentation (International Guide on Animal Biomedical Research and Ethical Code for Animal Experimentation of the Council for International Organization of Medical Sciences).

\section{Walking track analysis}

At three time points, 7, 15 and 21 weeks, rats $(n=5)$ were placed in a Perspex runway, as described by Westerga and Gramsbergen (1990). The lateral view of the animal was recorded directly with a video camera, whereas the ventral view of the animal was visualised by means of an adjustable mirror under the cage, positioned at an angle of $45^{\circ}$. In this manner, a split-screen image was obtained, with the lateral view of the rat in the upper half and the ventral view in the lower half (Meek 2000). The runway was illuminated with two 120-W concentric bulbs to improve contrast and to enhance the point of foot contact. Walking movements of the rat were recorded with a video camera containing a stroboscopic shutter ( 25 frames per second), creating blurfree stills for analysing the footsteps, until at least four 
consecutive and non-hesitant step cycles were collected (from 10 recordings). The videotape was then replayed frame by frame, until the maximal contact of the rat's foot to the floor was observed.

The videotaping technique was also used to obtain the stance factor, as described by Walker et al. (1994). The stance factor is the ratio between duration of floor contact (gait-stance duration) between the left (operated) and the right (non-operated) hind paw. Injured rats generally show a walking pattern with shorter gait-stance duration of the injured leg than of the non-injured leg.

\section{Withdrawal test}

At 7, 15 and 21 weeks ( $n=5$ for each time point), an electrostimulation test was carried out (in awake rats) over the whole sole using a bipolar electrode consisting of two copper wires. The lateral side of the left (operated) sole was stimulated proximally, distally and in between in all rats, as described by De Koning et al. (1986) and Meek et al. $(1997 ; 2003)$. A healthy rat immediately withdraws its foot and spreads its toes after stimulation. The threshold, that is, the lowest current causing this reflex, was recorded. Then, at each evaluation period, the sciatic nerve was cut in three rats and again the foot sole was electrically stimulated to verify the role of the sciatic nerve in the withdrawal test at the respective ages.

\section{Nerve processing}

At 7, 15 and 21 weeks ( $n=5$ for each time point), rats were sacrificed after dissecting the sciatic nerve implants with surrounding tissue from the muscle bed under deep anesthetised with $1 \%$ isoflurane (Forene, Abbott Laboratories Ltd., Queenborough, Kent, UK) and $\mathrm{O}_{2} / \mathrm{N}_{2} \mathrm{O}$. Immediately after explantation, conduits or conduit remnants were immersion-fixed in $2 \%(\mathrm{v} / \mathrm{v})$ glutaraldehyde in $0.1 \mathrm{M}$ phosphate buffer (pH 7.4) and embedded in Technovit 7100 (Kulzer, Wehrhein, Germany), or snap frozen in liquid nitrogen for light microscopy immunohistochemical evaluation ( $n=2$ for each time point). After Technovit (EBS, Connecticut) embedding semi-thin cross-sections $(3 \mu \mathrm{m})$ were stained with toluidine blue and examined with a light microscope. From the snap frozen samples, frozen sections $(7 \mu \mathrm{m})$ were cut at $-25^{\circ} \mathrm{C}$, fixed with acetone for $5 \mathrm{~min}$ and pre-incubated with PBS (NPBI, Emmercompascuum, The Netherlands) containing 10\% serum of the species that produced the primary antibodies diluted in PBS supplemented with 1\% (v/v) bovine serum albumin (BSA) for $60 \mathrm{~min}$ at room temperature. ED1 (Instruchemie, Hilversum, The Netherlands) recognises lysosomal antigens in macrophages and giant cells; HIS19 (DPC, Apeldoorn, The Netherlands) recognises mature membrane bound and intracellular major histocompatibility complex class II. Anti-collagen IV recognises human collagen IV and cross-reacts with rat and mouse collagen IV. The same holds for collagen III (both from South- ern Biotechnology Associates, ITK Diagnostics, Uithoorn, The Netherlands).

After washing, endogenous peroxidase was blocked by $0.1 \% \mathrm{H}_{2} \mathrm{O}_{2}$ and slides were incubated with secondary antibodies conjugated with horseradish peroxidase for $30 \mathrm{~min}$ at room temperature. Conjugates were diluted in PBS supplemented with $2 \%$ rat serum. Finally, after washing, the substrate 3-amino-9-ethylcarbazole was added, which turned into a red product by peroxidase activity. Sections were counterstained with hematoxilin, mounted with glycerine and analysed by light microscope image analysis (Leica Qwin, Leica Microsystems, Rijswijk, The Netherlands).

Statistical analysis: The data were tested for normal distribution. After this, subsequent non-parametric and parametric tests were performed, including ANOVA, KruskalWallis test, Tukey post hoc tests and Friedman two-way ANOVA. $P<0.05$ was accepted as a significant result. Controls are always the contralateral side of the same rat.

\section{RESULTS}

\section{Functional analysis}

In all groups, walking track patterns could be obtained. After 7, 15 and 21 weeks' recovery with the Hyaff1 1 nerve guide, rats in these groups all showed functional recovery of function of the sciatic nerve, since all rats were able to move and stretch the treated leg. Gait analysis of video recordings in the present study showed that walking remained disturbed up to 21 weeks. Outcome of the gait analysis is outlined in Figure 2. Normal toe spread was only observed in one rat at week 15 . The parameters walking on plantar side, absence of dragging, and hind feet placed within body contour showed the relative highest score at the three time points investigated, although walking on plantar side was slightly decreased at week 21 . A clear increase in performance through time was found in alternating steps, normal swing phase (swing is defined as fluent leg movement from behind to front, a 'circle-like' movement) and fluent walking. Absence of eversion and absence of exorotation exhibited a fluctuating pattern through time, with no clear signs of improvement.

\section{Sensory nerve recovery}

When stimulated with an electrical stimulus of $0.1 \mathrm{~mA}$ intensity at 7, 15 and 21 weeks, the unoperated leg could withdraw the foot and showed toe spreading. Results from the operated leg showed a different picture (Figure 3). After the operation, the maximum stimulus of $1.0 \mathrm{~mA}$ could not evoke any withdrawal, but $1.0 \mathrm{~mA}$ was inserted as maximum stimulus in the graph for reasons of comparison. At week 7, the threshold to elicit a withdrawal response had decreased to $0.42 \mathrm{~mA}$, but this value persisted up to 21 weeks, without any indications of a further increase in sensitivity. A Friedman two-way ANOVA revealed a 


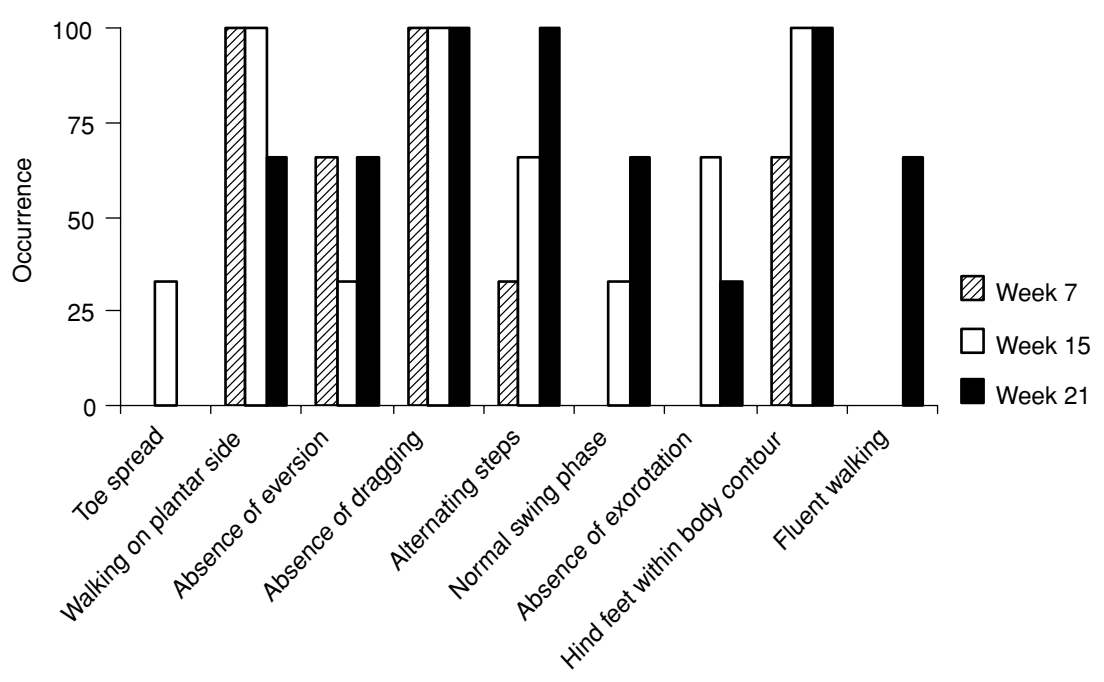

Figure 2 Histogram showing the percentages of rats with positive scores for each of the nine motoric behavioral parameters. For example, alternating steps is clearly improving in time.

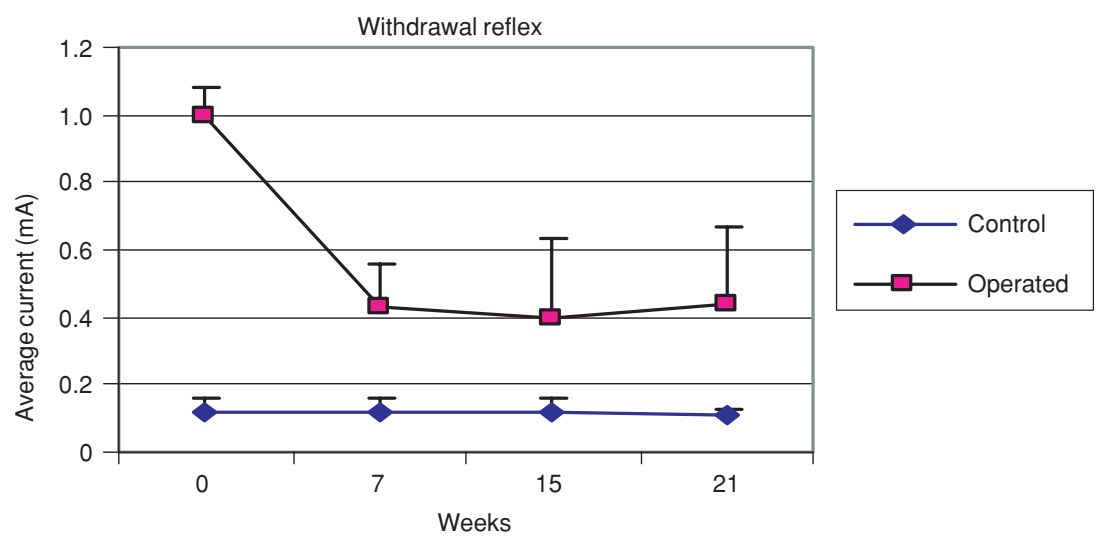

Figure 3 Graph showing the average current for a withdrawal reflex in control foot versus operated foot at time points $0,7,15$ and 21 weeks. The average current needed for a withdrawal reflex in the operated leg did decrease quickly to approximately $0.42 \mathrm{~mA}$ after 7 weeks, but remained stable up to 21 weeks. Friedman two-way ANOVA revealed a significant difference for stimulus strength throughout time in the operated side because of the decrease in stimulus from day 0 to week $7(P<0.05)$, but not for the control side $(P>0.05)$. There was no interaction effect between time and stimulus strength $(P>0.05)$.

significant difference for stimulus strength throughout time in the operated side due to the decrease in stimulus from day 0 to week $7(P<0.05)$, but not for the control side $(P>0.05)$. There was no interaction effect between time and stimulus strength $(P>0.05)$. Automutilation was detected in one rat at week 7 , but the incidence increased with time (Table 1).

\section{Stance factor}

Stance phase duration was calculated as the relative amount of floor contact time of the affected hind leg in relation to the non-affected hind leg (Dijkstra et al. 2000). The relative duration values increased in time from $44.7 \%$ (SEM = 13.2) at week 7 to $52.1 \%(\mathrm{SEM}=12.3)$ at week 15 , and up to $71.1 \%(\mathrm{SEM}=12.4)$ at week 21 . However, no statistical difference was found among the three groups (KruskalWallis, $P>0.05$ ).

\section{Histology}

After 7 weeks' regeneration of the sciatic nerve with the Hyaff11 conduit, transversal sections show that continuity of the regenerated nerve was re-established over the full length of the conduit [Figure 4(A) and (D)]. Regeneration and increase of nerve tissue diameter continued at 15 weeks [Figure 4(B) and (E)], up to 21 weeks [Figure $4(\mathrm{C})$ and $(\mathrm{F})]$. The orientation of the nerve tissue (fibers) was more linear at week 15 and 21 when compared to nerve tissue at week 7 [Figure 4(D), (E) and (F)]. Also, 

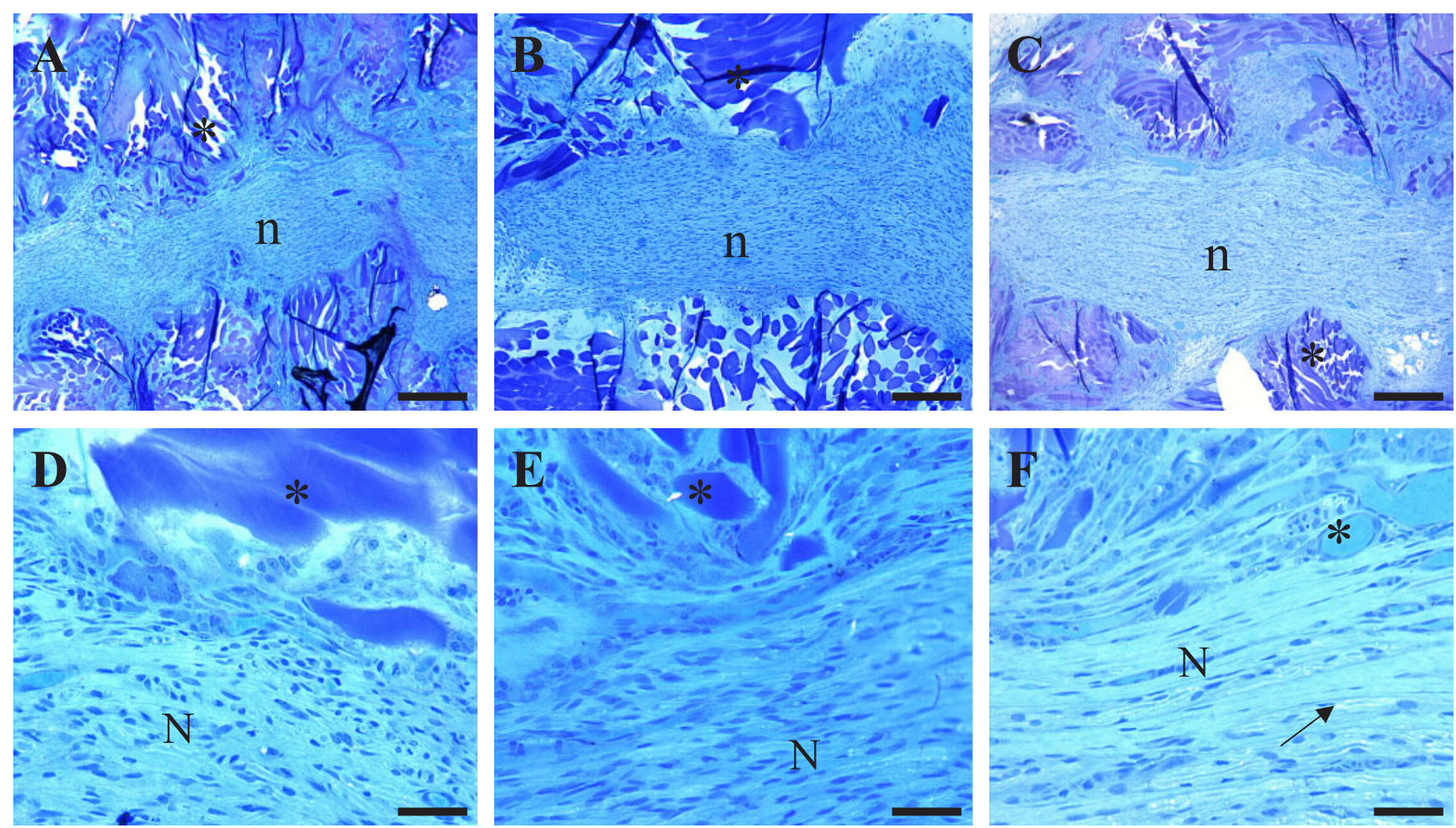

Figure 4 Light micrographs of transversal sections of the nerve guide explanted sections that were toluidine blue stained. (A), (B) and (C) represent week 7, 15 and 21, respectively. (D), (E) and (F) represent enlargements of biomaterial/nerve tissue interfaces for week 7,15 and 21 , respectively. $\mathrm{N}=$ nerve tissue; asterisks point at biomaterial; arrow in $(\mathrm{F})$ points at myelinated nerve tissue. Scale bars in (A), (B) and (C) represent $2 \mathrm{~mm}$; scale bars in (D), (E) and (F) represent $100 \mu \mathrm{m}$.

\section{Table 1 Hind paw automutilation in rats implanted with a Hyaff11 nerve guide}

\begin{tabular}{lllll}
\hline $\begin{array}{l}\text { Weeks after } \\
\text { implantation }\end{array}$ & Number of rats $(n)$ & No automutilation & $\begin{array}{l}\text { Moderate } \\
\text { automutilation }\end{array}$ & $\begin{array}{l}\text { Severe } \\
\text { automutilation }\end{array}$ \\
\hline 7 & 5 & 4 & 1 & 0 \\
15 & 5 & 0 & 2 & 3 \\
21 & 5 & 0 & 1 & 4 \\
\hline
\end{tabular}

many myelinated fibers were observed at week 21 , while such fibers were only sparsely observed at week 7 and 15.

Large amounts of biomaterial with surrounding foreign body reaction were found at all time points. The remnants of biomaterial were encircling the newly formed nerve tissue and were not observed within the nerve tissue itself. Foreign body reaction was also limited to the biomaterial and not observed in the nerve tissue (Figure 4). Large amounts of macrophages and giant cells were surrounding the biomaterial at all time points, as was confirmed with immunostainings specific for ED1 (lysosomal antigen specifically recognising macrophages and giant cells), applied to coronal sections (Figure 5). ED1 immunostainings show that presence of macrophages is not limited to the original outside and inside of the conduit wall at week 7, 15 and 21. At week 7 and 15, most macrophages are, however, found at the original rim position of the conduit while at some places a large influx of phagocytosing cells could be observed [Figure 5(A) and (B)]. At week 21, individual Hyaff11 fibers were completely surrounded by macrophages, while the tubular structure was lost. At all time points it was clear that the tubes had collapsed.

ED1 immunostaining demonstrated that many phagocytosing cells, like macrophages (and giant cells), did surround the tube walls and their remnants with increasing intensity. Stroma penetrated the tube via cracks in the tube wall and surrounded the newly formed nerve tissue at week 15 and 21. Immunostaining for collagen III and IV showed presence of these matrix proteins in and around the degrading structure of the tube and nerve tissue. Collagen III and IV were present at the original rim of the tube and on the inside of the place where the original lumen had been at week 7 [Figure 6(A) and (B)]. Nerve 

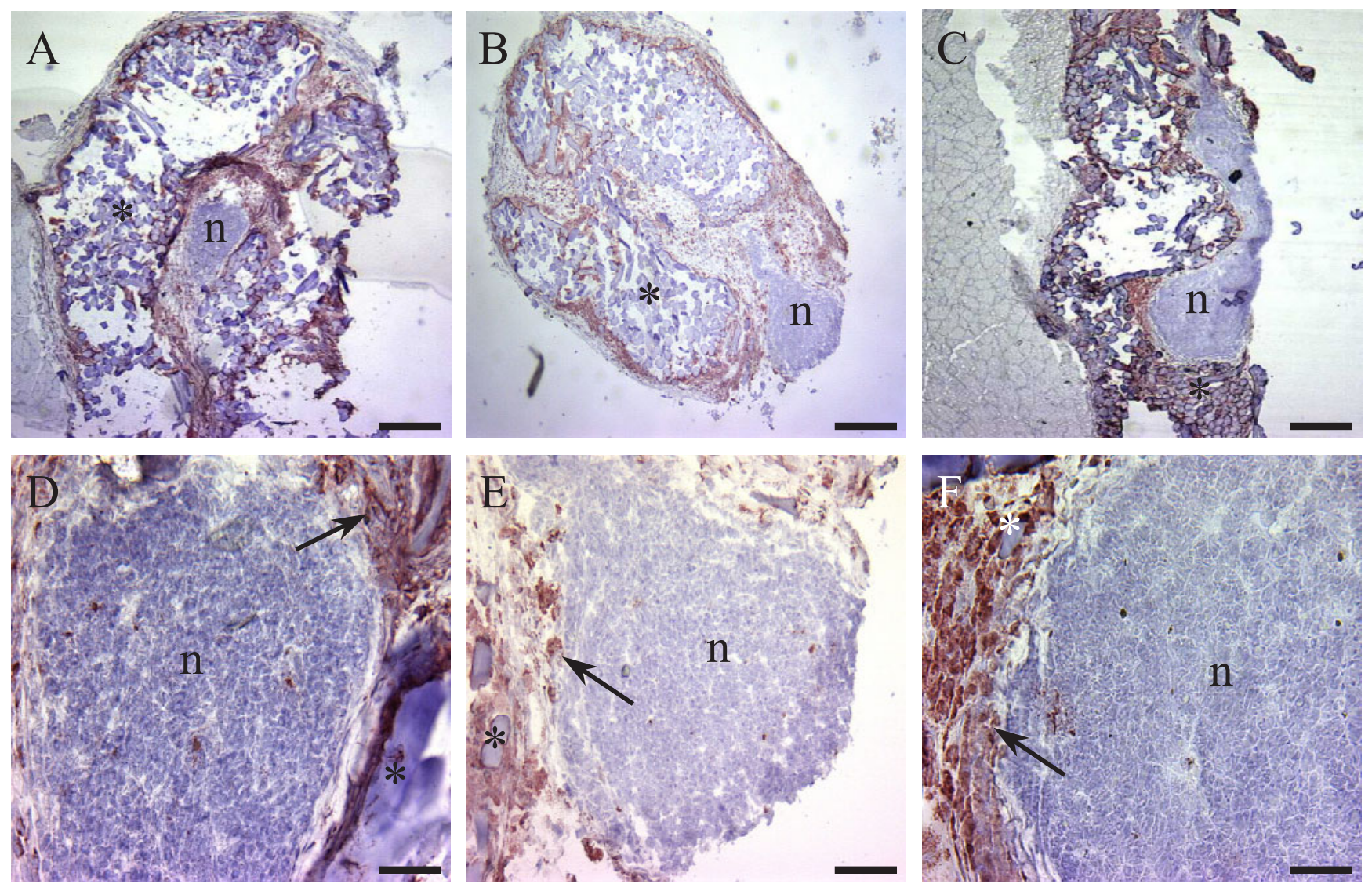

Figure 5 Light micrographs of coronal sections of the nerve guide explants immunocytochemically stained for ED1 (macrophages). (A), (B) and (C) represent week 7, 15 and 21, respectively. (D), (E) and (F) represent enlargements of biomaterial/nerve tissue interfaces for week 7,15 and 21 , respectively. $\mathrm{n}=$ nerve tissue; asterisks point at biomaterial; arrows point at ED1-immunopositive cells at the rim of the nerve tissue. Scale bars in (A), (B) and (C) represent $250 \mu \mathrm{m}$; scale bars in (D), (E) and (F) represent $40 \mu \mathrm{m}$.

tissue was therefore surrounded by both collagens. Collagen III and IV were also present at the influx of stroma, which also contained macrophages at week 21 [Figure $6(\mathrm{C})$ and (D)].

From the immunostained coronal sections $(n=5$ for each animal), the surface square area was determined, as well as the total surface area of tube material and nerve tissue together. This was done by using light microscope (Leica Qwin, Leica Microsystems, Rijswijk, The Netherlands) image analysis. This system differentiates between immunostained and non-stained parts of the image projected. By comparing the different surfaces at the same magnification, an indication of the change in surface area is obtained. The average percentages of nerve tissue surface areas were $10 \%( \pm 1.5 \%), 15 \%( \pm 1.7 \%)$ and $17 \%$ $( \pm 2.3 \%)$ for week 7,15 and 21 , respectively. The average percentage of nerve tissue areas differed significantly [Kruskal-Wallis, $F(2.34)=5.844 ; P<0.05]$. Post hoc tests showed a significant difference between week 7 and 15 [Tukey post hoc test $F(3.21)=3.956 ; \mathrm{P}<0.05$ ] and between week 7 and 21 [Tukey post hoc test $F(3.21)=$ 4.622; $P<0.05]$. No significant difference was found in the average percentage of nerve tissue surface areas between week 15 and 21 [Tukey post hoc test $F(3.21)=1.917$; $P>0.05]$.

\section{DISCUSSION}

The aim of this study was to assess functional nerve recovery as well as histological analysis of nerve outgrowth through a $1-\mathrm{cm}$ gap in the sciatic nerve of the rat with a Hyaff11-based nerve guide. It was shown that functional nerve repair could be established with a Hyaff11-based nerve guide since gait analysis showed that several functional parameters exhibited a clear increase in incidence through time while others were completely re-established when first measured at week 7 . Also, nerve sensory recovery was already re-established at week 7 , but pain withdrawal sensitivity did not increase through time and remained stable. This shows that return of pain withdrawal reflexes, although not optimal, occurs within a relatively short time frame. At the level of nerve tissue recovery, it was shown that regenerating axons had bridged the 1-cm gap within 7 weeks. Newly formed nerve tissue 

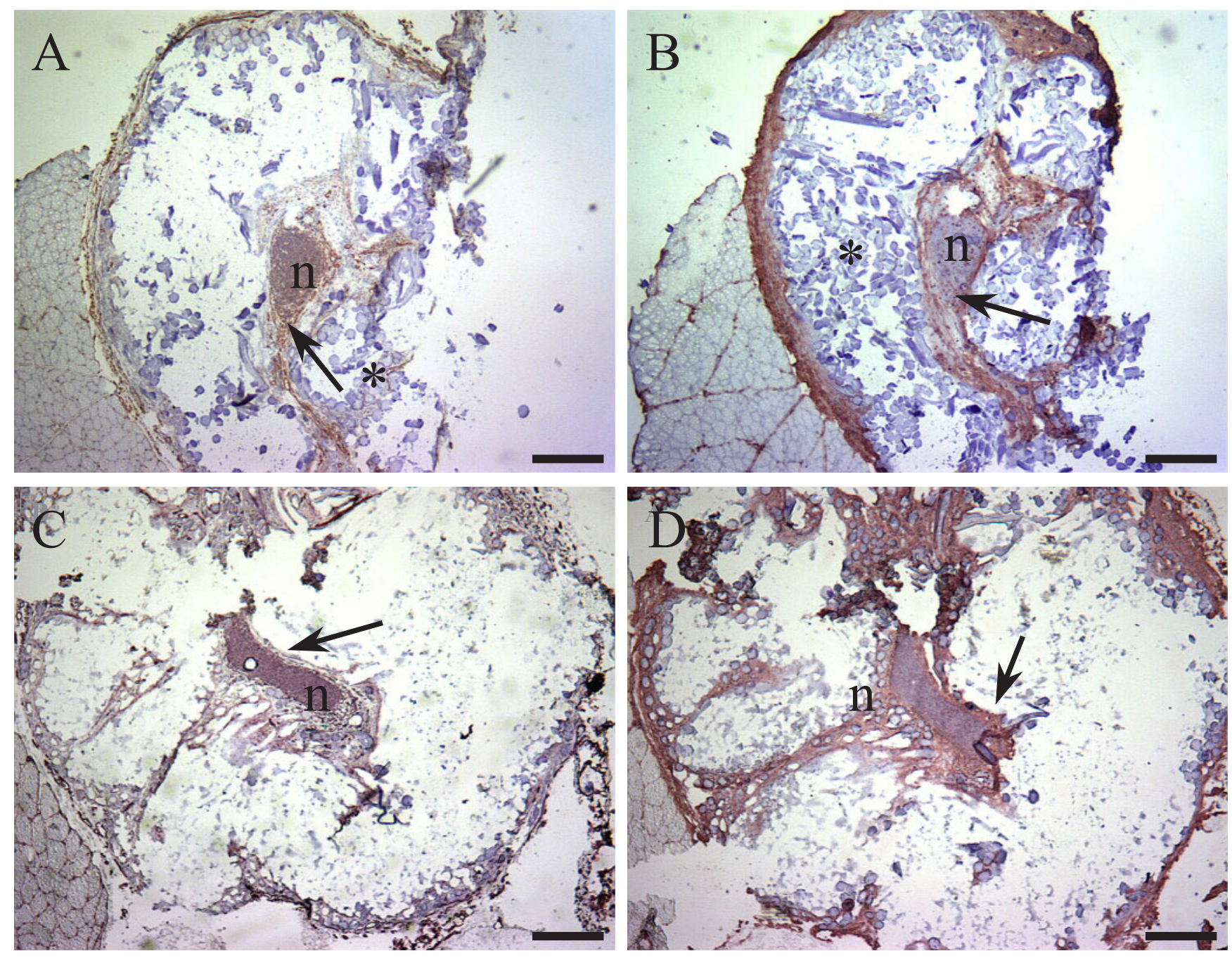

Figure 6 Light micrographs of coronal sections (obtained from the middle part) of the nerve guide explants immunocytochemically stained for collagen III (A and C) and IV (B and D). $\mathrm{n}=$ nerve tissue; asterisks point at biomaterial; arrows point at immunopositive staining at the rim of the nerve tissue. Scale bars represent $250 \mu \mathrm{m}$.

within the remnants of the conduit was observed in all animals at all time points. Although the tubes collapsed during the regeneration process, nerve tissue diameter did however increase with time, thereby forming a counterbalance against the slow decay of biomaterial and its remarkable degradation by which the lumen of the conduit is lost.

Functional peripheral nerve recovery has been described in literature (De Koning et al. 1986; Bain et al. 1989; Walker et al. 1996). Recovery with biodegradable poly(DL-lactide- $\varepsilon$-caprolactone) nerve guides has been described in detail by Meek (2000). In his studies he showed that the use of such thin-walled nerve guides results in a relatively good functional repair as described by gait analysis, reflex tests and nerve electrical activity (Meek $e t$ al. 2003). We too report conduit collapsing, but also functional repair. Collapsing of the tube was however expected on the basis of a biocompatibility/degradation study in which we implanted conduits subcutaneously in rats (unpublished data). Up to 3 weeks, no collapsing was observed but after that tube degradation, due to hydrolysis and tissue reactions, led to a cylindrical structure of biomaterial in which the lumen was completely replaced with the tube wall remnants. We assumed that the time frame of 3 weeks would be long enough for the regenerating axons to bridge the 1-cm gap before collapsing could possibly obstruct outgrowth. Here we have shown that this assumption was justified. Although the average nerve tissue diameter was fairly small after 7 weeks, it is clear that nerve fibers had bridged the gap. To prevent tube collapsing, thin-walled nerve conduits need mechanical (inside) support to achieve better functional nerve repair (Meek et al. 1996). Application of modified denaturated muscle tissue seems a promising perspective in animal nerve guide/repair studies (Glasby et al. 1986), especially in the view of bridging larger gaps. 
Pain withdrawal reflexes showed a strong increase in sensitivity in the first time period but remained stable at $0.43 \mathrm{~mA}$ afterwards. This result is not as good as was achieved with the poly(DL-lactide- $\varepsilon$-caprolactone nerve guide (Meek et al. 1997), but comparable with the result of another study of Meek et al. (1999), in which the thin walled nerve guide was filled with modified denaturated muscle tissue. Constriction of nerve tissue by the Hyaff11 material could explain why sensory function does not improve from week 7 to 21 . Whether at later time points a better sensory nerve recovery is achieved with the Hyaff11 nerve guide remains to be investigated. Altogether, we have seen that sensory and motoric functions of the rat hind leg can be restored with a Hyaff11based nerve guide, although functional recovery is not optimal.

One of the major components of fibrous tissue is collagen III, but collagen III also makes part of the epineurial membrane around nerve tissue as well as collagen IV (Rutka et al. 1988; Low and Moy 1992). Here we have seen that collagen III and IV formation occurred around the nerve tissue and that collagen III is strongly expressed around the remnants of biomaterial after 21 weeks. Furthermore, large amounts of macrophages, matrix and giant cells surround the biomaterial at week 21 , but few cells are present within the nerve tissue itself. Altogether, this could actually be a start for formation of the perineurium. In conclusion, we assume that further maturation of the nerve tissue will lead to a more substantial regenerated sciatic nerve with a decreasing tissue reaction since fewer and fewer remnants are expected to be present. To summarise, (1) the nerve construct in this study is not ideal but can be used to achieve peripheral nerve repair. (2) Long-term studies will eventually reveal whether a better functional recovery is achieved and to what extent tube material may hamper further nerve regeneration. (3) Further improvements on the Hyaff11 conduit design might lead to an optimal construct to bridge short nerve defects for clinical use.

\section{REFERENCES}

Andreassi L. 1995. Bioengineering in dermatology: General aspects and perspectives. Clin Dermatol, 13:369-73.

Avitabile T, Marano F, Castiglione F, et al. 2001. Biocompatibility and biodegradation of intravitreal hyaluronan implants in rabbits. Biomaterials, 22:195-200.

Bain JR, Mackinnon SE, Hunter DA. 1989. Functional evaluation of complete sciatic, peroneal and posterior tibial nerve lesions in the rat. Plast Reconstr Surg, 83:129-36.

Benedetti L, Cortivo R, Berti T, et al. 1993. Biocompatibility and biodegradation of different hyaluronan derivatives (Hyaff) implanted in rats. Biomaterials, 14:1154-60.

Campoccia D, Hunt JA, Doherty PJ, et al. 1996. Quantitative assessment of the tissue response to films of hyaluronan derivatives. Biomaterials, 17:963-75.
Davidson JM, Nanney LB, Broadley KN, et al. 1991. Hyaluronate derivatives and their application to wound healing: Preliminary observations. Clin Mater, 8:171-7.

De Koning P, Brakkee JH, Gispen WH. 1986. Methods for producing a reproducible crush in the sciatic and tibial nerve of the rat and rapid and precise testing of return of sensory function. 7 Neurol Sci, 74:237-46.

Den Dunnen WFA. 1996. Biodegradable nerve guides. PhD thesis, University of Groningen, The Netherlands.

Doolabh VB, Hertl MC, Mackinnon SE. 1996. The role of conduits in nerve repair: A review. Rev Neurosci, 7:47-84.

Galassi G, Brun P, Radice M, et al. 2000. In vitro reconstructed dermis implanted in human wounds: Degradation studies of the HA-based supporting scaffold. Biomaterials, 21:2183-91.

Glasby MA, Gschmeissner SE, Huang CLH, et al. 1986. Degenerated muscle grafts for peripheral nerve repair in primates. 7 Hand Surg (Br), 11:347-51.

Grigolo B, Roseti L, Fiorini M, et al. 2001. Transplantation of chondrocytes seeded on a hyaluronan derivative (Hyaff $\left.{ }^{\circledR}-11\right)$ into cartilage defects in rabbits. Biomaterials, 22:2417-24.

IJkema-Paassen J, Jansen K, Gramsbergen A, et al. 2004. Transection of peripheral nerves, bridging strategies and effect evaluation. Biomaterials, 25:1583-92.

Joshi HN, Stella VJ, Topp EM. 1992. Drug release from membranes of hyaluronic acid and its esters. $\mathcal{F}$ Control Release, 20:109-22.

Kyyronen K, Hume L, Benedetti L, et al. 1992. Methylprednisolone esters of hyaluronic acid in ophthalmic drug delivery: In vitro release study. Int $\mathcal{F}$ Pharmacol, 22:161-9.

Low SQ Moy RL. 1992. Scar wars strategies: Target collagen. $\mathcal{7}$ Dermatol Surg Oncol, 18:981-6.

Mohammad JA, Warnke PH, Pan YC, et al. 2000. Increased axonal regeneration through a biodegradable amnionic tube nerve conduit: Effect of local delivery and incorporation of nerve growth factor/hyaluronic acid media. Ann Plast Surg, 44:59-64.

Meek MF, Den Dunnen WFA, Schakenraad JM, et al. 1996. Evaluation of functional nerve recovery after reconstruction with a poly(DL-lactide- $\varepsilon$-caprolactone) nerve guide, filled with modified denaturated muscle tissue. Microsurgery, 17:555-61.

Meek MF, Den Dunnen WFA, Bartels HL, et al. 1997. Peripheral nerve regeneration and functional nerve recovery after reconstruction with a thin-walled biodegradable poly(DL-lactide- $\varepsilon$-caprolactone) nerve guide. Cell Mater, $7: 53-62$.

Meek MF, Dijkstra JR, Den Dunnen WFA, et al. 1999. Functional assessment of sciatic nerve reconstruction: Biodegradable poly(DLLA- $\varepsilon-C L)$ nerve guide versus autologous nerve grafts. Microsurgery, 19:381-8.

Meek MF. 2000. Artificial nerve guides: Assessment of nerve function. PhD thesis, University of Groningen, The Netherlands.

Meek MF, Van der Werff JF, Klok F, et al. 2003. Functional nerve recovery after bridging a $15 \mathrm{~mm}$ gap in rat sciatic nerve with a biodegradable nerve guide. Scand $\mathcal{F}$ Plast Reconstr Surg Hand Surg, 37:258-65.

Rutka JT, Apodaca G, Stern R, et al. 1988. The extracellular matrix of the central and peripheral nervous systems: Structure and function. $\mathcal{F}$ Neurosurg, 69:155-70.

Seckel BR, Jones D, Hekimian KJ, et al. 1995. Hyaluronic acid through a new injectable nerve guide delivery system 
enhances peripheral nerve regeneration in the rat. $\mathcal{f}$ Neurosci Res, 40:318-24.

Walker JL, Evans JM, Meade P, et al. 1994. Gait-stance duration as a measure of injury and recovery in the rat sciatic nerve model. 7 Neurosci Methods, 52:47-52.
Wang KK, Nemeth IR, Seckel BR, et al. 1995. Hyaluronic acid enhances peripheral nerve regeneration in vivo. $\mathcal{F}$ Neurosci Res, 15:318-24.

Zacchi V, Soranzo C, Cortivo R, et al. 1998. In vitro engineering of human skin-like tissue. $\mathcal{F}$ Biomed Mater Res, 40:187-94. 

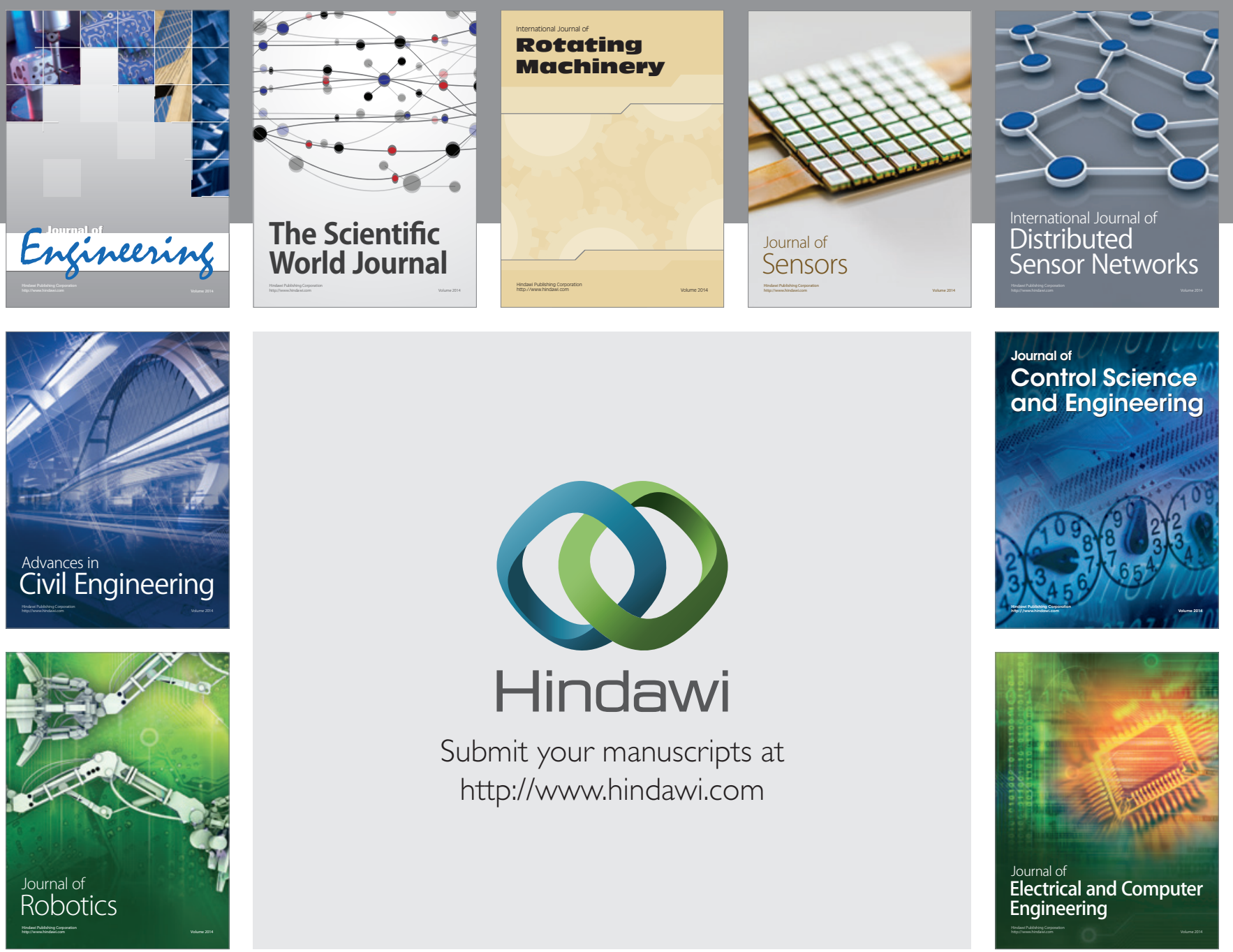

Submit your manuscripts at

http://www.hindawi.com
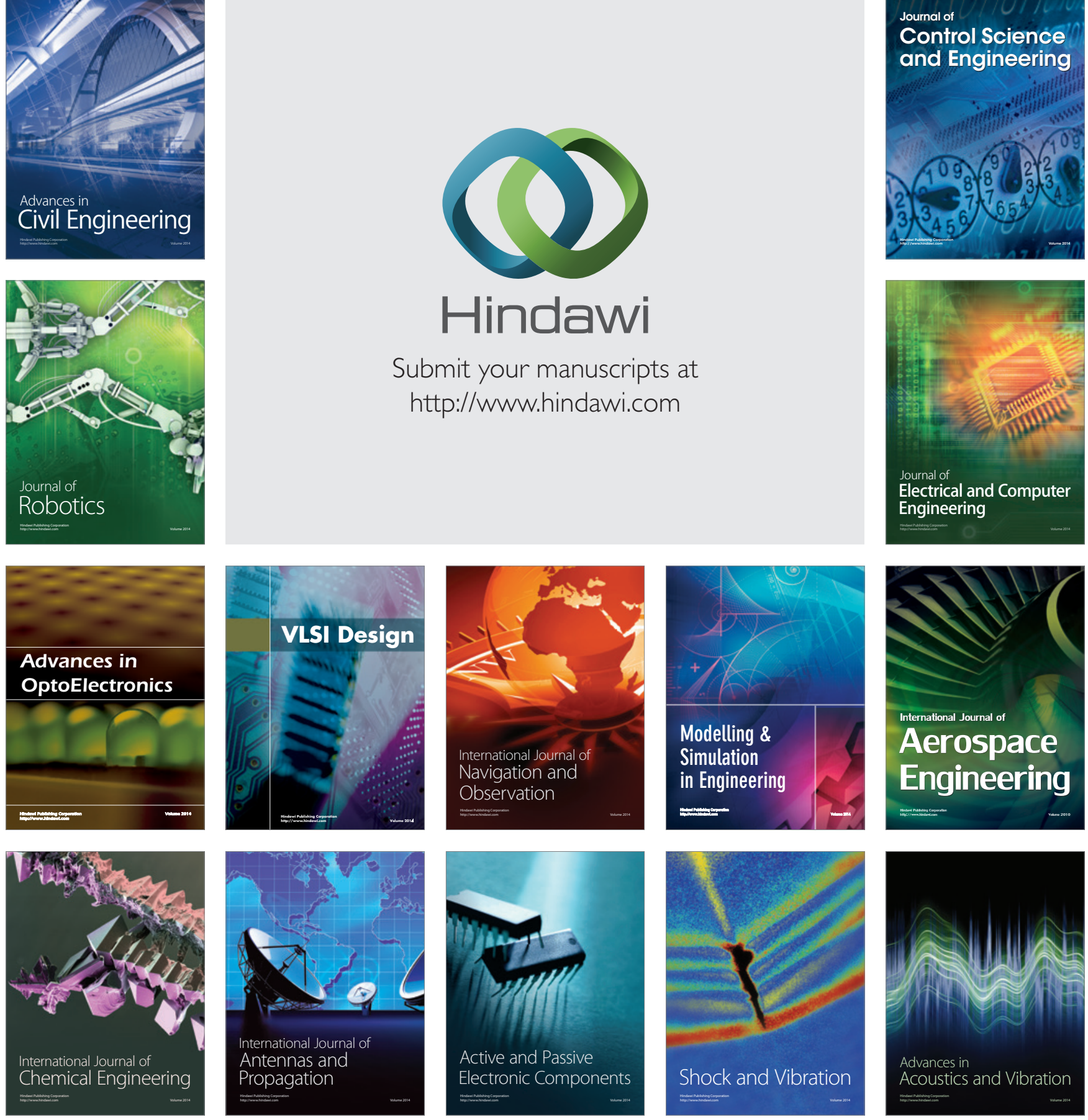